\title{
Synthesis of Dependent Multichannel ECG using Generative Adversarial Networks
}

\author{
Eoin Brophy \\ INFANT Research Centre and School of Computing, Dublin City University \\ Ireland
}

\begin{abstract}
Access to medical data is highly regulated due to its sensitive nature, which can constrain communities' ability to utilise these data for research or clinical purposes. Common de-identification techniques to enable the sharing of data may not provide adequate protection for an individual's personal data in every circumstance. We investigate the ability of Generative Adversarial Networks (GANs) to generate realistic medical time series data to address these privacy and identification concerns. We generate synthetic, and more significantly, multichannel electrocardiogram (ECG) signals that are representative of waveforms observed in patients. Successful generation of high-quality synthetic time series data has the potential to act as an effective substitute for actual patient data. For the first time, we demonstrate a multivariate GAN architecture that can successfully generate dependent multichannel time series signals. We present the first application of multivariate dynamic time warping as a means of evaluating generated GAN samples. Quantitative evidence demonstrates our GAN can generate data that is structurally similar to the training set and diverse across generated samples, all whilst ensuring sufficient privacy guarantees for the underlying training data.
\end{abstract}

\section{CCS CONCEPTS}

\section{- Computing methodologies $\rightarrow$ Machine learning.}

\section{KEYWORDS}

Multivariate time series synthesis, Generative Adversarial Networks, Medical data synthesis

\section{ACM Reference Format:}

Eoin Brophy. 2020. Synthesis of Dependent Multichannel ECG using Generative Adversarial Networks. In Proceedings of the 29th ACM International Conference on Information and Knowledge Management (CIKM '20), October 19-23, 2020, Virtual Event, Ireland. ACM, New York, NY, USA, 4 pages. https://doi.org/10.1145/3340531.3418509

\section{PROBLEM}

Restrictions on the use and sharing of highly sensitive medical data represents a significant challenge in modern clinical research and development. In addition to traditional modes of data sharing, new machine learning techniques which rely on access to increasingly large datasets to train artificial intelligence systems present an

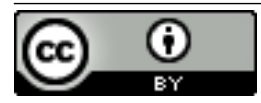

This work is licensed under a Creative Commons Attribution International 4.0 License.

CIKM '20, October 19-23, 2020, Virtual Event, Ireland

(C) 2020 Copyright held by the owner/author(s).

ACM ISBN 978-1-4503-6859-9/20/10.

https://doi.org/10.1145/3340531.3418509 extra layer of obstacles for researchers and clinicians to navigate. Addressing these obstacles can give rise to ethical issues, such as how we control these artificial intelligence systems and how data can be used while ensuring privacy is protected and public trust is maintained.

Practitioners with access to sought after data often find themselves working through complex regulatory frameworks and discovering that sharing and publishing the information available via the data to be extremely challenging. For example, personal sensitive data such as medical data intended for use in secondary purposes like clinical training or research requires anonymisation following its approval for dissemination.

Common methods for the de-identification of data do not guarantee privacy protection of all subjects in the dataset as it is possible to re-identify individuals [2]. This may result in the inability to share data with other research or clinical institutions. To compound these matters, there is often a shortage of available training data for clinicians and researchers alike significantly impeding scientific progress.

Generation of synthetic data is one such solution to the presented problem. The goal here lies in producing synthetic physiological data that is representative of real data gathered during the data collection experiment. It is important to note that substantial amounts of data are required to successfully train deep learning models for this purpose. Protecting the privacy of the underlying real dataset must also be observed and presents a difficult challenge to those concerned $[8,9]$.

If these problems can be addressed and overcome the generated data can then be published without breaching privacy and used in further training and research. Increasing the access to this type of data will encourage scientific studies and facilitate the upskilling of clinicians which will in turn aid in preventing or limiting chronic illnesses. This can contribute to a shift in the treatment paradigm from reactive to preventative healthcare.

In this paper, for the first time, we demonstrate the contributions of our method in generating realistic, dependent, multivariate physiological signals while maintaining sufficient levels of privacy in the training dataset.

\section{STATE OF THE ART}

Many early methods of physiological time series generation require expert domain knowledge to generate synthetic data. More recent developments in the machine learning space help to remove this dependency. A significant breakthrough in the generation of synthetic data was facilitated by the introduction of Generative Adversarial Networks (GANs). A GAN consists of a generator and a discriminator. The generator $G$ is a neural network that takes

Supervised By: Prof. Maarten De Vos, Prof. Geraldine Boylan and Prof. Tomás Ward. 
random noise $z \in \mathbb{R}^{r}$ and generates synthetic data. The discriminator $D$ is a neural network which determines if the generated data is real or fake. The generator aims to maximise the failure rate of the discriminator while the discriminator aims to minimise it. The two networks are locked in a two-player minimax game defined by the value function $V(G, D)(1)$, where $D(\boldsymbol{x})$ is the probability that $x$ comes from the real data rather than the generated data [5].

$$
\begin{aligned}
\min _{G} \max _{D} V(G, D)=E_{x \sim p_{\text {data }}(x)}[\log D(\mathbf{x})]+ \\
E_{z \sim p_{\mathbf{Z}}(z)}[\log (1-D(G(\mathbf{z})))]
\end{aligned}
$$

GANs are an alternative method of generating synthetic data which do not require input from domain experts. They were first proposed in the seminal paper by Goodfellow in 2014 [5]. A recurrent GAN (RGAN) was first proposed in 2016 where the generator contained a recurrent feedback loop which used both the input and hidden states at each time step to generate the final output [7]. Recurrent GANs often utilise Long Short-Term Memory neural networks (LSTMs) in their generative models to avoid the vanishing gradient problem [6].

While the focus to date has been on the development of GANs for improved image generation, there has been a movement towards the use of GANs for time series generation and more specifically physiological time series generation. We are aware of only one work that implements both a GAN and a conditional GAN (CGAN) to generate real-valued multi-dimensional medical time series data [3]. More recent attempts to generate synthetic physiological data used bidirectional LSTMs in the generator and convolutional neural networks in the discriminator [12]. While these works are focused on the generation of medical data, they generate independent, single-channel time series data. Extending on this, we develop a GAN architecture for dependent multivariate medical time series generation.

Also, there is a lack of consensus in current research on the best choice of evaluation metric for GANs [1]. We propose a new solution and therefore assess the generated multichannel time series data using a novel application of multivariate Dynamic Time Warping (DTW) to determine similarity measures of the dependent generated signals to the dependent multichannel training samples.

\section{APPROACH}

In this section, we present our MV-GAN (Multivariate GAN) model for generating synthetic, dependent, multivariate physiological time series data. Structurally, our model builds on architectures of previous works. We increase the limited sequence length of 30 shown in [3] to a length of 187 . This yields a time series more representative of digitised ECG for the time windows considered. This length is arbitrary and can be varied with the discriminator architecture to produce data sequences of differing length. In terms of generating multichannel data, we increase the number of features available at the input and output of our model which enables it to learn the characteristics of the training set and generate multivariate data, this has not been done in previous works. Extending on the previous models, we also implement 2-dimensional convolutionpooling layers and include a minibatch discrimination layer in the discriminator in an effort to prevent mode collapse. The optimiser also has noise introduced to its gradients to create a differentially private MV-GAN model (MV-GAN-DP). The architecture of the generator and discriminator models are displayed in Figure 1 and 2 respectively.

The generator consists of a two-layer stacked LSTM with 50 hidden units in each layer and a fully connected layer at the output.

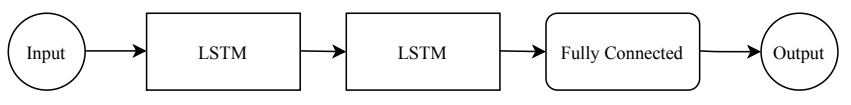

Figure 1: Block Diagram of Generator Architecture

The discriminator is a four-layer 2-dimensional convolutional neural network, a minibatch discrimination layer was added to prevent mode collapse. Noise was added to the gradient of the optimiser to ensure differential privacy for the MV-GAN-DP model.

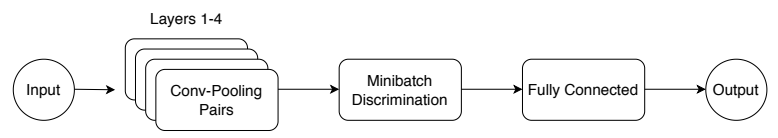

Figure 2: Block Diagram of Discriminator Architecture

\section{METHODOLOGY}

\subsection{Dataset}

The publicly available MIT-BIH Arrhythmia dataset on PhysioNet was used for the experiments in this paper $[4,10]$. This database contains 48 half-hour long recordings of two-channel ambulatory ECG. Figure 3 shows the lead configuration with an example trace of a classic ECG expected from the leads.

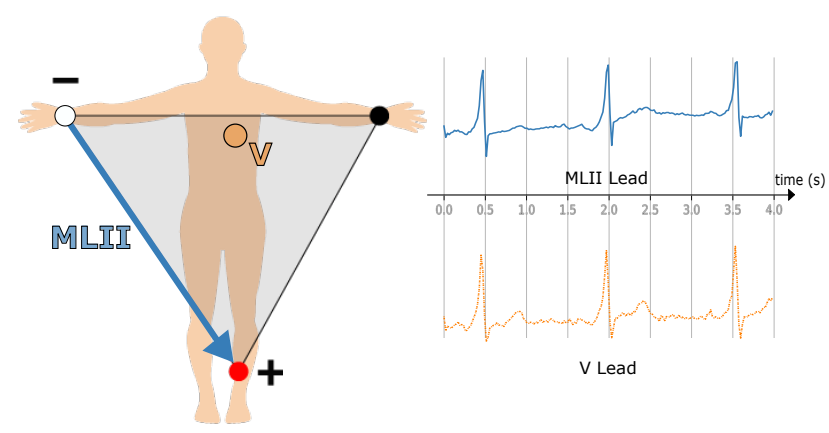

Figure 3: Lead configuration used for that data collection experiment with corresponding expected ECG trace

4.1.1 Data Preprocessing. The dataset was pulled from PhysioNet and loaded using Python's wfdb library. Before training our MVGAN, the dataset required preprocessing in the form of R-peak alignment, segmentation and downsampling. An example of the multichannel input data is shown in Figure 3. 


\subsection{Training}

The MV-GAN was trained for 50 epochs with a batch size of 50 multivariate samples. The discriminator was trained multiple times for a single generator training phase as recommended by [5]. RMSprop optimizer was used with learning rate $\alpha=0.0002$ as it is computationally efficient and works well for this deep learning model. The MV-GAN was first trained without minibatch discrimination (MBD). Next, a minibatch discrimination layer was added to the discriminator with the number of outputs set to $3,5,8$ and 10 to determine how the number of outputs affects the data generated. Noise was also introduced into the gradients of the discriminator optimiser to ensure a differentially private network [11].

\subsection{Evaluation}

4.3.1 Quality. To assess the quality of generated data from our MV-GAN, the Maximum Mean Discrepancy (MMD) and multivariate Dynamic Time Warping was computed against the training set. MMD is used here to reinforce the DTW results and to demonstrate that the GAN iteratively learns and generates data from a distribution that is more representative of the training data distribution. Multivariate, dependent DTW $\left(D T W_{D}\right)$ was calculated to determine similarity measures across the dependent signals in the generated data against the training data. To our knowledge, this is the first time this method is used to evaluate generated data from time series GANs.

4.3.2 Privacy. To assess presence disclosure, a membership inference attack was run. The sample size $r$ was varied between $[1000,10000]$ training records while the threshold $\epsilon$ ranged from $[0.05,0.5]$ of the mean Euclidean distance between all samples. A synthetic dataset of 1000 generated samples was used for this test. Presence disclosure occurs if it is possible to determine that a particular record was used to train a GAN by observing the generated samples.

\section{RESULTS}

In this section we focus on training and the data generated by the MV-GAN without differential privacy unless explicitly stated otherwise. From Figure 4 it is apparent that the GAN with MBD layer 10 did not converge and it is retained here to emphasise our opinion regarding the difficulty in developing a useful GAN design capable of stable training. However, the qualitative results for the other models appear to have successfully converged and are capable of generating realistic, multivariate and dependent ECG data. The inclusion of the MBD layers introduces a degree of variety into the generated data samples. Without the MBD layers, there is zero variation across the generated samples which demonstrates that mode collapse can be prevented with the addition of these layers.

An example of real training data versus generated data is shown in Figure 5. To the best of our knowledge, this is the first instance of multivariate physiological signal with dependencies present that has been generated using GANs. An offset on the V lead is introduced for visualisation purposes. However, this cannot be solely considered as an evaluation of GAN performance due to the lack of a suitable metric to measure data quality. We address this challenge in the following section.
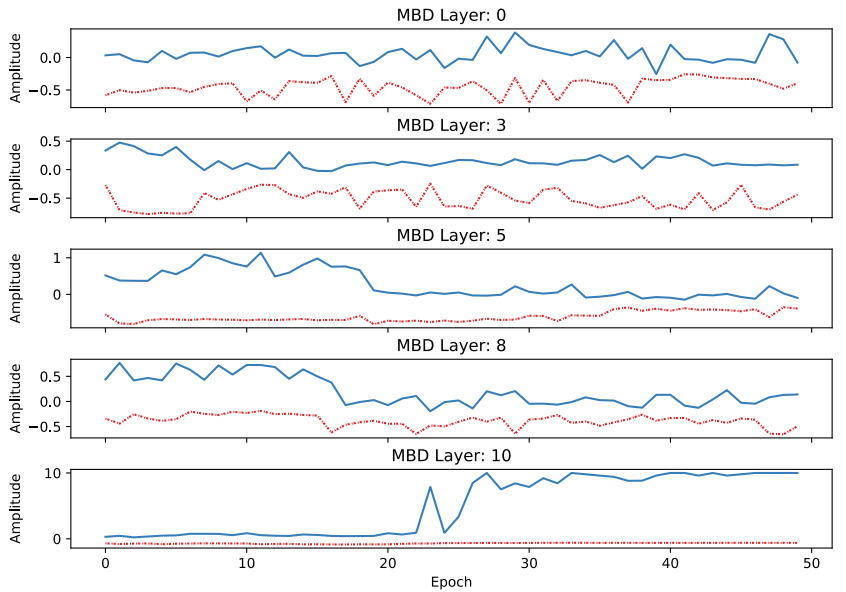

Figure 4: Training losses of MV-GAN across different MBD layers, D(Blue) G(Dashed-Red)
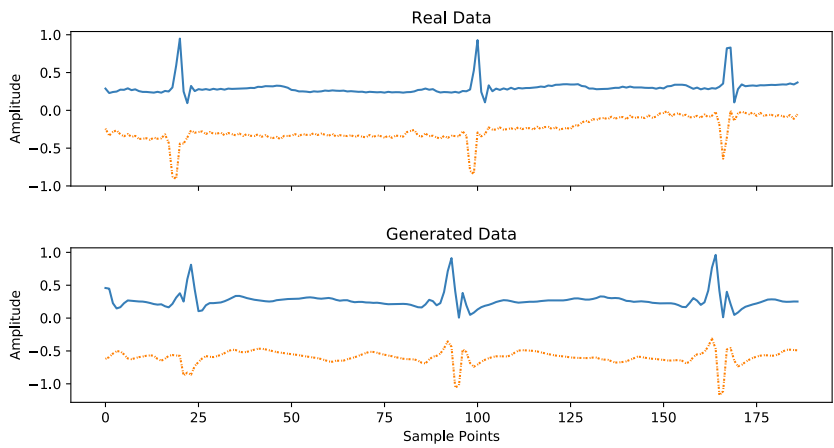

Figure 5: Real training ECG data (top) vs. Generated ECG data from MV-GAN (bottom)

\subsection{Evaluation}

5.1.1 Quality. Here, we augment our visual and qualitative assessment through the development of suitable objective quantitative metrics. MMD results in Figure 6 demonstrate that as the GAN iterates through the training process it is generating data from a distribution that is more representative of the training data distribution. The MMD values for generated data (solid-blue) is contrasted with a random noise sample (dashed-red). In all cases, with one exception (MBD 10), the MMD for our generated samples reduces over the training process.

Results for DTW extended to multivariate time series are plotted in Figure 7. It can be seen that as the MV-GAN iterates through its training process the $D T W_{D}$ values decrease for the respective MBD layers, excluding MBD 10 and MBD 0. However, the DTW values in MBD 0 are consistently low compared to all other layers. Hence, we can say that the distance measures between the dependent generated signals and the dependent training signals are reducing throughout the training process, indicating that our MV-GAN has successfully captured the multivariate training data distribution. 

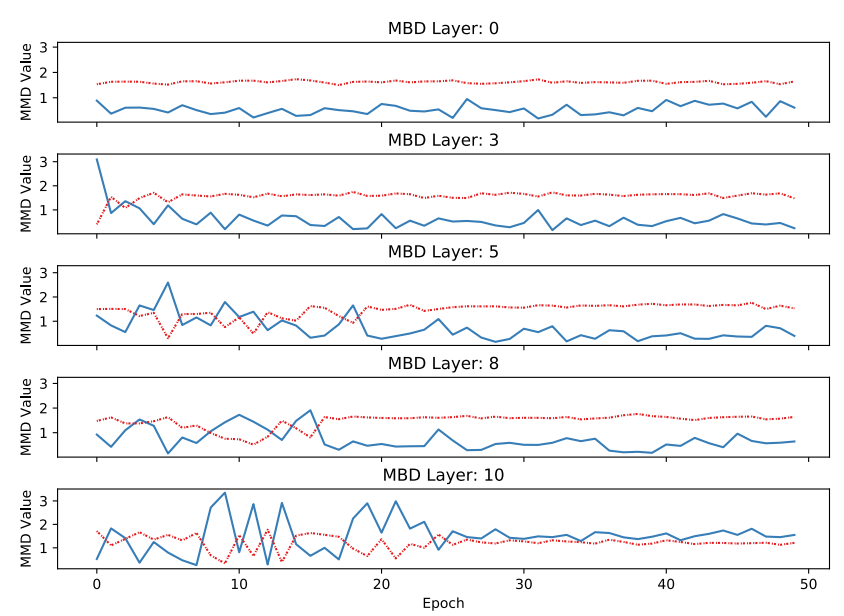

Figure 6: Calculated MMD for each MBD layer at the end of every epoch. Blue line shows the data distribution between training and generated data. Dashed-red line represents the distribution between random noise and the training data.

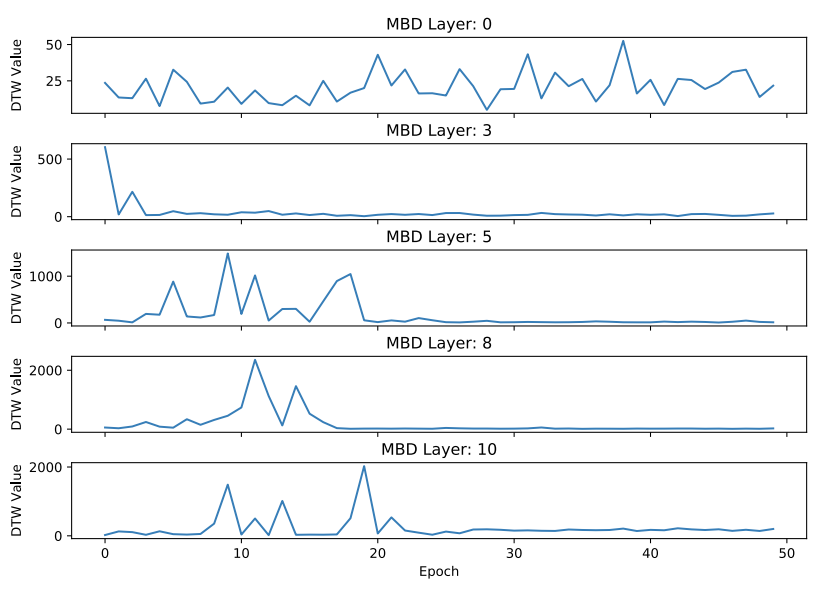

Figure 7: $D T W_{D}$ for each MBD layer, calculated at the end of each epoch, shows similarity between the multivariate training and generated data.

5.1.2 Privacy. The number of training records identified is very low (Recall), with approximately $0 \%$ correctly identified for $\epsilon \leq 0.4$ $*$ mean distance. However, as the threshold $\epsilon$ increases above this boundary, the number of records correctly identified as training samples increases independent of the sample size $r$ for the MVGAN without differential privacy. The MV-GAN-DP preserves the training data privacy with no training records identified for $\epsilon \leq$ $0.5 *$ mean distance. Precision is approximately $100 \%$ for all $\epsilon$ and $r$, which means once an attacker deems that a sample originates from the training set it is almost always correctly attributed to the training set. Overall, this result tells us that these GAN architectures can generate data similar in distribution to the training set while maintaining sufficient privacy of the data.

\section{CONCLUSION AND FUTURE WORK}

The MV-GAN proposed in this work has demonstrated the capability of generating high-quality, dependent, multichannel ECG signals. Our introduction of an MBD layer in the GAN architecture leads to a more robust design which avoids mode collapse and results in the generation of the desired diverse multichannel time series signals. We have also introduced a new quantitative method for assessment of output quality for multichannel time series GANs, namely $D T W_{D}$. With the addition of a differentially private MVGAN architecture we can also generate data that does not violate the privacy of the training data.

Multivariate time series data presents an opportunity for the application of GANs in tackling the data shortage and sharing problem in medical research. In terms of our motivating challenge and future work, successfully capturing and generating diverse samples of multichannel and dependent physiological data means we have the potential to use this technology for such a use case and in particular for clinical training and research applications. With that goal in mind, we have shown, for the first time, a GAN design capable of generating high-quality dependent multichannel physiological time series with quality similar to that present in clinically relevant data repositories.

\section{REFERENCES}

[1] Antonia Creswell, Tom White, Vincent Dumoulin, Kai Arulkumaran, Biswa Sengupta, and Anil A. Bharath. 2018. Generative Adversarial Networks: An Overview. IEEE Signal Processing Magazine 35, 1 (Jan 2018), 53-65. https: //doi.org/10.1109/MSP.2017.2765202 arXiv:cs.CV/1710.07035

[2] Khaled El Emam, Elizabeth Jonker, Luk Arbuckle, and Bradley Malin. 2011. A systematic review of re-identification attacks on health data. PLoS ONE 6 (2011), 12. https://doi.org/10.1371/journal.pone.0028071

[3] Cristóbal Esteban, Stephanie L. Hyland, and Gunnar Rätsch. 2017. Real-valued (Medical) Time Series Generation with Recurrent Conditional GANs. https: //doi.org/10.1002/fut arXiv:1706.02633

[4] A. L. Goldberger, L. A. N. Amaral, L. Glass, J. M. Hausdorff, P. Ch. Ivanov, R. G. Mark, J. E. Mietus, G. B. Moody, C.-K. Peng, and H. E. Stanley. 2000. PhysioBank, PhysioToolkit, and PhysioNet: Components of a New Research Resource for Complex Physiologic Signals. Circulation 101, 23 (2000), e215-e220. Circulation Electronic Pages: http://circ.ahajournals.org/content/101/23/e215.full PMID:1085218; doi: 10.1161/01.CIR.101.23.e215.

[5] Ian Goodfellow, Jean Pouget-Abadie, Mehdi Mirza, Bing Xu, David Warde-Farley, Sherjil Ozair, Aaron Courville, and Yoshua Bengio. 2014. Generative Adversarial Nets. In Advances in Neural Information Processing Systems 27. Curran Associates, Inc., 2672-2680. http://papers.nips.cc/paper/5423-generative-adversarial-nets. pdf

[6] Sepp Hochreiter and Jj Urgen Schmidhuber. 1997. Lstm. Neural Computation 9, 8 (1997), 1735-1780. https://doi.org/10.1162/neco.1997.9.8.1735 arXiv:1206.2944

[7] Daniel Jiwoong Im, Chris Dongjoo Kim, Hui Jiang, and Roland Memisevic. 2016. Generating images with recurrent adversarial networks. arXiv:1602.05110 http://arxiv.org/abs/1602.05110

[8] Scott McLachlan, Kudakwashe Dube, and Thomas Gallagher. 2016. Using the CareMap with Health Incidents Statistics for Generating the Realistic Synthetic Electronic Healthcare Record. Proceedings - 2016 IEEE International Conference on Healthcare Informatics, ICHI 2016 (2016), 439-448. https://doi.org/10.1109/ ICHI.2016.83

[9] Patrick E. McSharry, Gari D. Clifford, Lionel Tarassenko, and Leonard A. Smith. 2003. A dynamical model for generating synthetic electrocardiogram signals. IEEE Transactions on Biomedical Engineering 50, 3 (2003), 289-294. https://doi. org/10.1109/TBME.2003.808805

[10] G. B. Moody and R. G. Mark. 2001. The impact of the MIT-BIH arrhythmia database. IEEE Engineering in Medicine and Biology Magazine 20, 3 (2001), 45-50. https://doi.org/10.1109/51.932724

[11] Liyang Xie, Kaixiang Lin, Shu Wang, Fei Wang, and Jiayu Zhou. 2018. Differentially Private Generative Adversarial Network. arXiv:1802.06739 http: //arxiv.org/abs/1802.06739

[12] Fei Zhu, Fei Ye, Yuchen Fu, Ouan Liu, and Bairong Shen. 2019. Electrocardiogram generation with a bidirectional LSTM-CNN generative adversarial network. Scientific Reports 9, 1 (2019), 1-11. https://doi.org/10.1038/s41598-019-42516-z 